\title{
Numerical simulation of earth fissures due to groundwater withdrawal
}

\author{
Z. Wang ${ }^{1}$, Y. Zhang ${ }^{1}$, J. Wu ${ }^{1}$, J. Yu ${ }^{2}$, and X. Gong ${ }^{2}$ \\ ${ }^{1}$ School of Earth Sciences and Engineering, Nanjing University, Nanjing, 210093, China \\ ${ }^{2}$ Key Laboratory of Earth Fissures Geological Disaster, Ministry of Land and Resources of China, \\ Geological Survey of Jiangsu Province, Nanjing, 210049, China
}

Correspondence to: Y. Zhang (zhangyun@nju.edu.cn)

Published: 12 November 2015

\begin{abstract}
Excessive groundwater withdrawal can cause land subsidence and earth fissures. The initiation and propagation of earth fissures are related to tensile failure and crack propagation in soils. Based on fracture mechanics, the crack band model (CBM), one of the smear crack models which is relatively easy to construct and convenient to be integrated into standard finite element codes is used in this paper. The calculated results of CBM are less dependent on the sizes of finite elements. The model was applied to simulate the formation and propagation of earth fissures in the hydrostratigraphic units with a bedrock ridge. The simulated positions and patterns of earth fissures coincide with field observations, suggesting that the modeling approach is adept to simulate the initiation and propagation of earth fissures due to groundwater withdrawal.
\end{abstract}

\section{Introduction}

Discontinuous problems may arise when performing numerical simulation analysis on earth fissures. It is rather inefficient to treat a discontinuity as an internal boundary when applying a standard finite element method (FEM), especially when formation and propagation of fissures are concerned. This approach usually requires intense work in order to deal with the constantly changing shape and topology of the discontinuity. New methods have been presented aiming to solve such discontinuous problems, such as the extended finite element method (XFEM) (Khoei et al., 2014), the element-free Galerkin method (EFGM) (Belytschko et al., 1995), the discrete element method (DEM) (Camones et al., 2013), the displacement discontinuous method (DDM) (Detournay and Gordeliy, 2011). Most of them behave reasonably well in some particular circumstances but they show limitations in other cases because of their different design nature. On the other hand, it would be quite convenient if a similar approach would be compatible with existing standard finite element methods. The smeared cracking model (SCM) was presented by Rashid (1968), aiming to adapt the standard finite element analysis where instances of discontinu- ous problems are present. It is relatively easy to build from an existing standard FEM and has been proved successful in describing mode-I fracture behavior. In addition, the method is widely-used in many fracture mechanics field such as concrete fracturing (Li et al., 2007). Early SCM implementations used stress as a failure criterion, which leads to a serious mesh-dependency (Cedolin and Bazant, 1980). One substantial modification has been made since the energy-based criterion from fracture mechanism was considered. Softening process now has been incorporated instead of a pure brittle failure. One typical case is the Crack Band Model (CBM) proposed by Bazant and Oh (1983), which will be applied to simulate earth fissures in this paper.

\section{Crack band model}

Cracking within an element is considered as a three-stage process in the crack band model: intact stage, micro-fissure stage and cracked stage (Fig. 1). The critical strains between these three stages are denoted as $\varepsilon_{0}$ and $\varepsilon_{f}$, which are determined by Eqs. (1) and (2). 


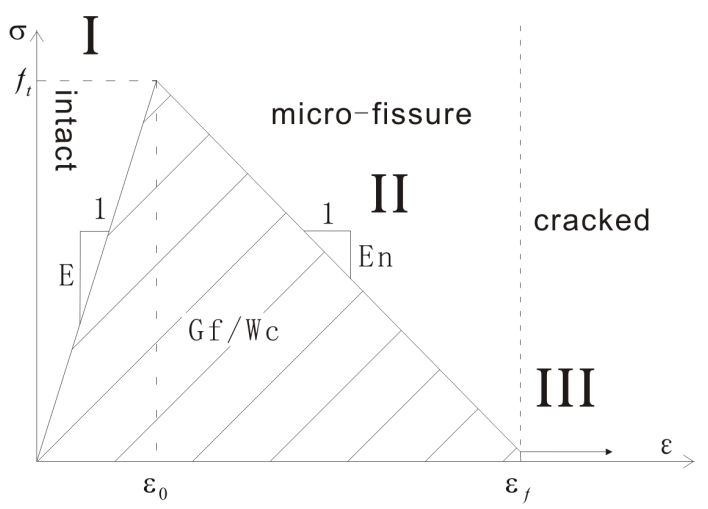

Figure 1. Constitutive relation in CBM.

$\varepsilon_{0}=\frac{f_{\mathrm{t}}}{E}$

$\varepsilon_{f}=\frac{2 G_{f}}{f_{\mathrm{t}} w_{c}}$

where $E$ is the tensile modulus, $\mathrm{kPa} ; f_{\mathrm{t}}$ is the tensile strength, $\mathrm{kPa} ; G_{f}$ is the release energy rate, $\mathrm{J} \mathrm{m}^{-2}$; and $w_{c}$ is the element size, $\mathrm{m}$.

When an element is deemed as a cracked or micro-fissure element, this element is treated as mechanical anisotropy. A reduced modulus perpendicular to crack face is then used in order to decrease the stress with an increasing strain. Based on addition of strain which means that the total strain is the sum of the crack strain and the material strain, the constitutive relation is modified from

$\Delta \sigma=\left[\mathbf{D}^{\mathrm{co}}\right] \Delta \varepsilon$

to (Rots, 1991)

$\Delta \boldsymbol{\sigma}=\left[\mathbf{D}^{\mathrm{co}}-\mathbf{D}^{\mathrm{co}} \mathbf{N}\left(\mathbf{D}^{\mathrm{cr}}+\mathbf{N}^{T} \mathbf{D}^{\mathrm{co}} \mathbf{N}\right)^{-1} \mathbf{N}^{T} \mathbf{D}^{\mathrm{co}}\right] \Delta \boldsymbol{\varepsilon}$

where $\Delta \sigma$ is the stress increment; $\Delta \boldsymbol{\varepsilon}$ is the strain increment; $\mathbf{N}$ is the coordinate-transformation matrix from global coordinate system to local coordinate system in front of a crack tip; $\mathbf{D}^{\text {co }}$ is the modulus matrix of intact material under the global coordinate system; $\mathbf{D}^{\text {cr }}$ is the modulus matrix under the local coordinate system with its $x$ coordinate coincident with the crack surface, which contains an element modulus normal to the crack surface and a modulus tangent to the crack surface, and it is given as

$\mathbf{D}^{\mathrm{cr}}=\left[\begin{array}{ll}E_{\mathrm{n}} & 0 \\ 0 & E_{s}\end{array}\right]$

where $E_{\mathrm{n}}$ is the modulus normal to the crack face, and $E_{S}$ is the modulus tangent to the crack face.
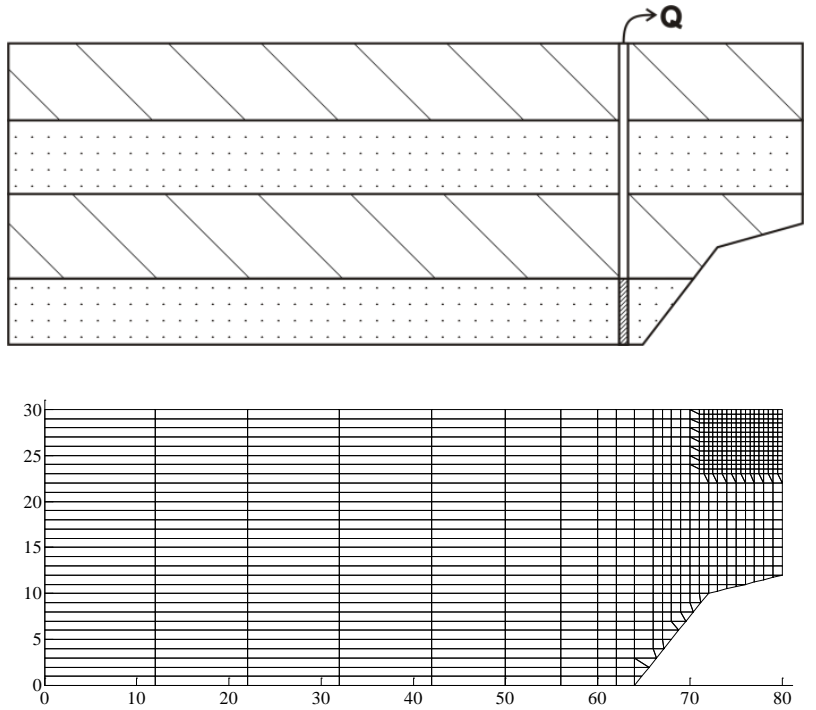

Figure 2. Conceptual model (top panel) and the finite element mesh (bottom panel) of the earth fissure example.

\section{Simulation on pumping-reduced earth fissure formation}

The non-tectonic formation and propagation of earth fissures are often considered to be closely related to groundwater extraction activities. This is clearly the case in Suzhou-WuxiChangzhou (SXC) area in Jiangsu Province, China (Zhang et al., 2008; Wu et al., 2003). A simulation is performed based on the crack band model, aiming to find the mechanism of earth fissure formation and propagation under the aquifer system with a bedrock ridge.

According to the local geological conditions and the current developmental state of earth fissures in the SXC area (Zhang et al., 2008), we can then propose a conceptual model (Fig. 2) assuming a plane strain problem. The depth of the whole Quaternary strata is $30 \mathrm{~m}$. Four layers are considered of alternating clay soil and sandy soil beds. More specifically, the first layer (on the top surface) and the third one are clay soil; and the other two beds correspond to the sandy soil. The irregular shape on the lower right corner of the conceptual model represents the bedrock ridge, which is cutting off the third and the fourth layers at depth (Fig. 2, top panel). The left boundary is set as a boundary of fixed water level with no excess pore pressure and no horizontal displacement. Finally, other boundaries are assumed impervious. Pumping activities are assumed to lie on both sides of the bedrock ridge at the fourth layer with a constant flow rate $\left(100 \mathrm{~m}^{3} \mathrm{day}^{-1}\right)$. The phreatic level is $5 \mathrm{~m}$ below the ground surface and remains unchanged during the whole simulation because it is unexploited. The upper part of strata where earth fissures usually occur lies above the phreatic level. It can be considered as a linear elastic material when subjected to a tensile load because the soil in the upper part is often over-consolidated 


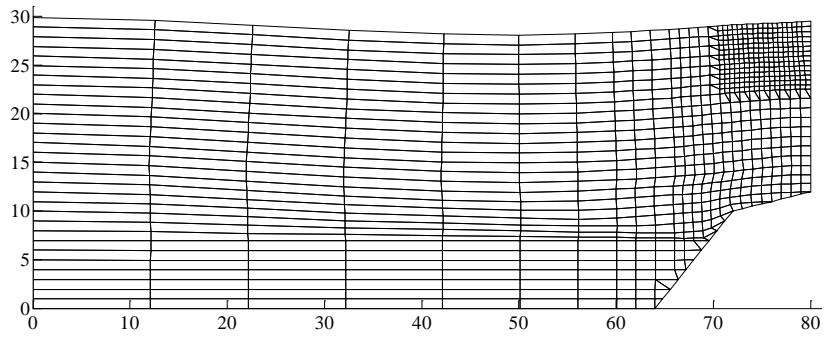

Figure 3. Deformation after consolidation.

with low confining pressure and unsaturation. Therefore, the constitutive relation is chosen to be the Duncan-Chang model under compressive conditions and linear-elasticity under tensile conditions.

Considering its symmetry, the simulation is conducted on the left part, as shown in the conceptual model in Fig. 2 (top panel). Calculation is carried out following the Biot's consolidation equations. Since fluid-solid coupling is concerned, it is notable that the pore pressure should be calculated in an incremental form instead of total form as given in Eq. (6)

$$
\left[\begin{array}{ll}
\overline{\mathbf{K}} & \mathbf{K}^{\prime} \\
\mathbf{K}^{\prime T} & \widetilde{\mathbf{K}}
\end{array}\right]\left\{\begin{array}{c}
\Delta \boldsymbol{\delta} \\
\Delta \boldsymbol{\beta}
\end{array}\right\}=\left\{\begin{array}{c}
\boldsymbol{R}-\boldsymbol{R}_{t}-\mathbf{K}^{\prime} \boldsymbol{\beta}_{\mathrm{t}} \\
\Delta t \boldsymbol{Q}-\widetilde{\mathbf{K}} \boldsymbol{\beta}_{\mathrm{t}}
\end{array}\right\}
$$

where $\overline{\mathbf{K}}$ is the element stiffness matrix; $\widetilde{\mathbf{K}}$ is the seepage matrix; $\mathbf{K}^{\prime}$ is the coupled matrix; $\Delta \boldsymbol{\delta}$ is the incremental displacement; $\Delta \beta$ is the incremental pore pressure; $\boldsymbol{R}$ is the load; $\boldsymbol{R}_{t}$ is the load already balanced by stress; $\Delta t$ is the time interval; $\boldsymbol{Q}$ is the flow at nodes; $\boldsymbol{\beta}_{\mathrm{t}}$ is the total pore pressure in the last time interval which equals zero in the first time step.

The strata deformation after pumping is indicated in Fig. 3. When the pumping started, pore pressure at depth decreased, forming a zone with negative excess pore pressure, which was centered at the pumping point. Effective stress increased accordingly, which has caused a land subsidence bowl on top of the pumping well. It should be noted that the main deformation occurs at the deep clay soil.

Figure 4 shows the minor principal stress distribution as well as earth fissure formation and propagation at different time during pumping. The red zone represents the tensile zone and the black lines within represent the earth fissure simulated by the crack band model. Firstly, a self-weighting consolidation simulation is performed to determine the initial stress distribution instead of simply assuming a $k_{0}$ consolidation state, as in Fig. 4a. The self-weight in all elements is not applied simultaneously but activated layer by layer from bottom to top, aiming to represent the deposition process. It can be observed that the stress distribution yielded due to the self-weighting consolidation is slightly different from a simple $k_{0}$ consolidation state. The bedrock ridge induces a decrease in the upper layers in the minor principal stress. When pumping activity is initiated, the minor principal stress in the soil at depth increases significantly. Moreover, maximum
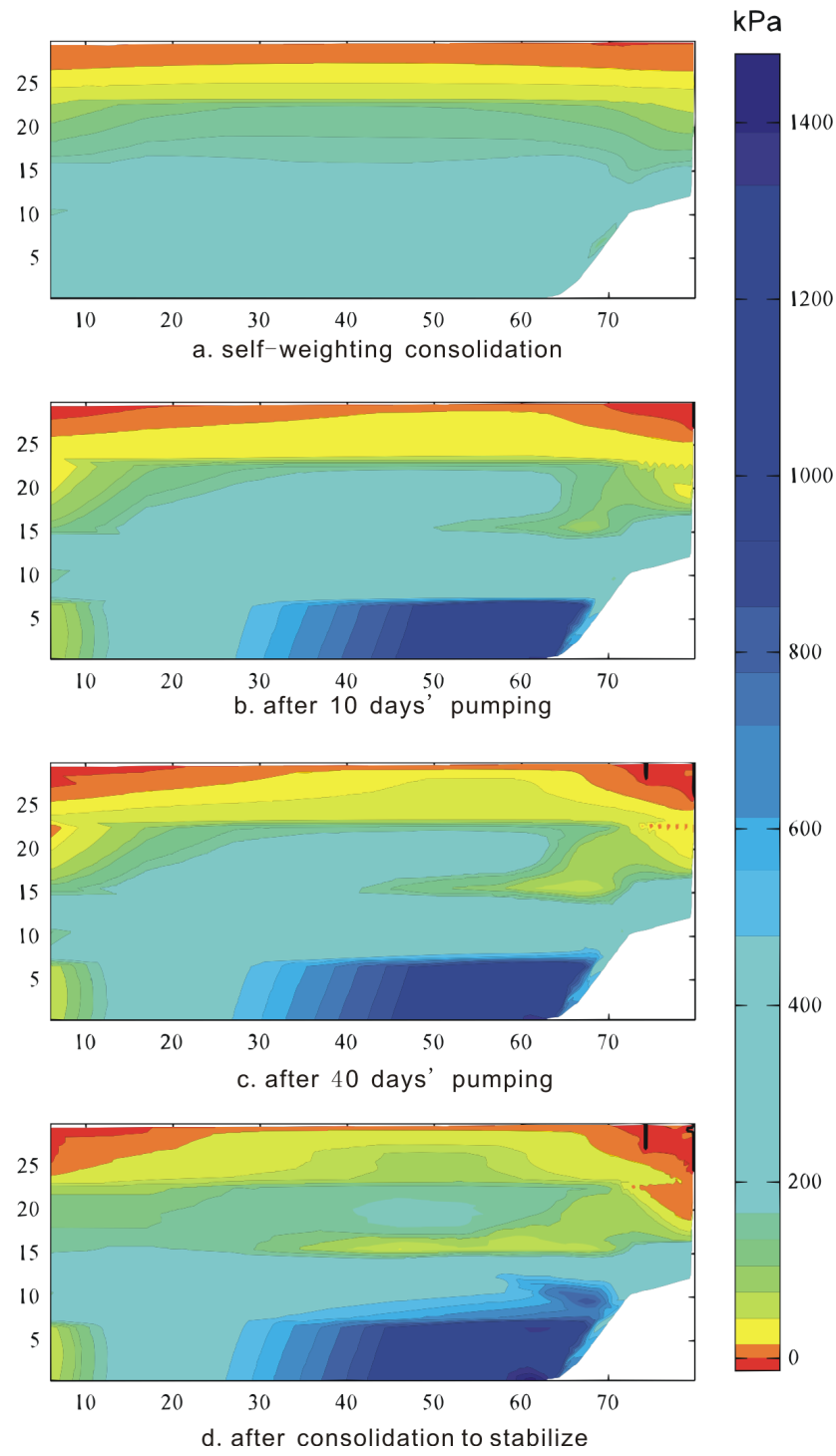

Figure 4. Distribution of minor principal stress during pumping.

change in stresses occurs near the pumping well. Meanwhile, the minor principal stress above the bedrock ridge begin to decrease, the tensile zone emerges and extends constantly. After 10 days of pumping, an earth fissure has occurred at the very top of the bedrock ridge, which was propagating downward (Fig. 4b). After a 40-day pumping period, a second crack parallel to the main crack was formed (Fig. 4c). It is noted that after each earth fissure formation or propagation, the tensile zone shrinks towards the active crack horizontally but grows deeper at the front of the active crack tip because of the release of unbalanced stresses. This becomes clearer when the crack reaches a larger length. So activities of each crack are tending to impede nearby its crack and promote it (i.e. the crack). After the pumping period is ended, the tensile zone became even narrower but deeper than that at the beginning of the pumping period. It can be observed that, in 
the second layer, a tensile zone is also formed (Fig. 4d). Minor principal stress in this area is even smaller than the adjacent area above and is formed because of the discrepancy of displacement between the two different layers with contrasting mechanical characteristics. The simulation results are in good agreement with the field observations, indicating that it is suitable to use the crack band model to simulate earth fissure formation and propagation.

\section{Conclusions}

This paper discusses the simulation of earth fissuring using the crack band model. The simulation results indicate that the crack band model is useful to model earth fissures generation. An example of earth fissure formation and propagation is simulated based on the crack band model under the usual geological conditions with bedrock ridges in the $\mathrm{Su}-$ $\mathrm{Xi}-\mathrm{Chang}$ area of Jiangsu Province, China.

Acknowledgements. This paper was financially supported by the Ministry of Land and Resources of China (grant no. 20141109603), the National Nature Science Foundation of China (grant no. 41572250), and UNESCO and IUGS (no. 641).

\section{References}

Bazant, Z. P. and Oh, B. H.: Crack band theory for fracture of concrete, Mater. Struct., 16, 155-177, 1983.

Belytschko, T., Lu, Y. Y., and Gu, L.: Crack propagation by element-free Galerkin methods, Eng. Fract. Mech., 51, 295-315, 1995.
Camones, L. A. M., Vargas Jr., E. A., Figueiredo, R. P., and Velloso, R. Q.: Application of the discrete element method for modeling of rock crack propagation and coalescence in the step-path failure mechanism, Eng. Geol., 153, 80-94, 2013.

Cedolin, L. and Bazant, Z. P.: Effect of finite element choice in blunt crack band analysis, Comput. Meth. Appl. Mech. Eng., 24, 305316, 1980.

Detourna, E. and Gordeliy, E.: Displacement discontinuity method for modeling axisymmetric cracks in an elastic half-space, Int. J. Solids Struct., 48, 2614-2629, 2011.

Khoei, A. R., Vahab, M., Haghighat, E., and Moallemi, S.: A mesh-independent finite element formulation for modeling crack growth in saturated porous media based on an enriched-FEM technique, Int. J. Fract., 188, 79-108, 2014.

Li, Q., Zhang, B., Yu, Y., and Wang, J.: Numerical simulation of the process of hydraulic fracturing in earth and rockfill dams, Chinese J. Geotech. Eng., 29, 212-217, 2007.

Rashid, Y. R.: Ultimate strength analysis of pre-stressed concrete pressure vessels, Nucl. Eng. Design, 7, 334-344, 1968.

Rots, J. G.: Smeared and discrete representations of localized fracture, Int. J. Fract., 51, 45-59, 1991.

$\mathrm{Wu}, \mathrm{Z}$., Yu, Q., and Zhang, Y.: Forming process of earth fissure hazard in the Suzhou-Wuxi-Changzhou area, Hydrogeol. Eng. Geol., 30, 67-71, 2003.

Zhang, Y., Xue, Y., Wu, J., Yu, J., Wei, Z., and Li, Q.: Land subsidence and earth fissures due to groundwater withdrawal in the Southern Yangtse Delta, China, Environ. Geol., 55, 751-762, 2008. 